\title{
Optical Magnetoelectric Effect in Multiferroic Materials: Evidence for a Lorentz Force Acting on a Ray of Light
}

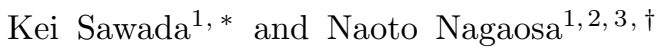 \\ ${ }^{1}$ Department of Applied Physics, the University of Tokyo, \\ 7-3-1, Hongo, Bunkyo-ku, Tokyo 113-8656, Japan \\ ${ }^{2}$ Correlated Electron Research Center (CERC), National Institute of Advanced Industrial \\ Science and Technology (AIST), Tsukuba Central 4, Tsukuba 305-8562, Japan \\ ${ }^{3}$ CREST, Japan Science and Technology Agency (JST), Japan
}

(Dated: November 10, 2018)

\begin{abstract}
We theoretically propose that the optical analog of a Lorentz force acting on a ray of light is realized in multiferroic materials such as $\mathrm{GaFeO}_{3}$ showing the magnetoelectric effect. The toroidal moment $\vec{T}=\sum_{j} \vec{r}_{j} \times \vec{S}_{j}$ plays the role of a "vector potential" while its rotation corresponds to a "magnetic field" for photons. Hence, the light is subject to the Lorentz force when propagating through the domain wall region of the ferromagnetic or ferroelectric order. A realistic estimate on the magnitude of this effect is given.
\end{abstract}

PACS numbers: 78.20.Ls, 42.70.Qs, 78.20.Fm

The analogy between optics and particle dynamics has been a guiding principle in physics even leading to wave mechanics, i.e., quantum mechanics. In the classical theory, their analogy is established through the variational principle, which gives the Newtonian equation of motion for a particle and the eikonal equation of light. The variational principle for a classical particle is given by $\delta \int v d s=0$ with the velocity $v=\sqrt{2[E-U(x)] / m}(E$ : total energy, $U(x)$ : potential energy, $m$ : mass of the particle). In geometrical optics, an equation for the trajectory of light is derived from the Fermat's principle; i.e., the time required to propagate from one to the other points is minimized. $\delta \int d s / V=\delta \int d s n(\vec{r}) / c=0$, where $V=c / n$ is the velocity of light in the medium of refractive index $n(\vec{r})$. This leads to the equation for the trajectory of light, which is similar to the equation of motion for a particle, as

$$
\frac{d}{d s}\left[n(\vec{r}) \frac{d \vec{r}}{d s}\right]=\operatorname{grad} n(\vec{r}) .
$$

In analogy to particle mechanics, we can interpret the above equation for light as follows. The term in the lefthand side is an "acceleration term". On the right-hand side, the gradient of the refractive index $\nabla n(\vec{r})$ acts as the "force" on the light and distorts the trajectory, giving rise to the refraction of light, i.e., Snell's law. Therefore $n(\vec{r})$ is regarded as the "scalar potential". In particle mechanics, there is another type of force, i.e., the Lorentz force, in the presence of the magnetic field. This force is the transverse one to the velocity of the particle and, historically, has led to the concept of the "field" and relativistic mechanics. Then the natural question to ask is whether the analogous force exists for light. It has been believed that there is no optical analog of magnetic field [1] 2], namely a Lorentz force on light in real space. On the other hand, a Lorentz force in $k$-space was introduced recently in a polarization-dependent equation of motion as a generalization of the geometrical optics 3 . This "Lorentz force" in momentum space is described by a Berry phase in analogy to an anomalous Hall effect [3] or a Magnus effect [4]. Haldane and Raghu focus on an edge state in a magnetic photonic crystal on the analogy of the quantum Hall effect [5]. The edge state is a polarization-dependent one-way waveguide with broken time-reversal symmetry. However the Lorentz force on light in real space has been missing up to now.

In this Letter, we theoretically propose that the Lorentz force on light independent of its polarization is realized in the multiferroic materials showing the nonreciprocal optical effect. The polarization-independent non-reciprocal optical effect requires a breaking of both inversion and time-reversal symmetries, which allows polarization-independent directional birefringence, called a magneto-chiral (MC) effect [ 6] 7] or an optical magnetoelectric (OME) effect [8] 9]. The difference between the $\mathrm{MC}$ and OME effects lies in their crystal structureswhether a magnet has a helical structure or a polar structure. These effects have directional dependence, namely, $\hat{k}$ dependence in optical response. We focus on magnets with a polar structure, namely, multiferroics. Novel optical properties in multiferroics are characterized by a toroidal moment $\vec{T} \equiv \sum_{i} \vec{r}_{i} \times \vec{S}_{i}$ [10], where $\vec{r}_{i}$ and $\vec{S}_{i}$ are the displacement of the center position from atoms and a spin of an electron at $i$ th site, respectively. We note that the definition of the toroidal moment implies that even in a antiferroelectric and antiferromagnetic system, the toroidal moment can be non zero. In ferroelectric and ferromagnetic systems, we can rewrite the toroidal moment as $\vec{T} \propto \vec{P} \times \vec{M}$, where $\vec{P}$ and $\vec{M}$ are spontaneous electric polarization and magnetization, respectively. Hereafter we assume that multiferroics are simultaneously ferroelectric and ferromagnetic for simplicity. We can reverse the toroidal moment by an external field by reversing the electric polarization or the magnetization. The toroidal 
moment brings about the OME effect that refractive index depends on whether the propagation direction is parallel or antiparallel to the toroidal moment. The OME effect is expressed as $n_{\rightarrow}-n_{\leftarrow} \propto \hat{k} \cdot \vec{T}$, where arrows in the subscripts represent directions of light and $\hat{k} \equiv \frac{\vec{k}}{|\vec{k}|}$.

We demonstrate below a novel refraction phenomenon caused by an inhomogeneous toroidal moment in multiferroics. The equation of motion for light in multiferroics contains a Lorentz force in real space, where a spatially inhomogeneous toroidal moment gives rise to a magnetic field for light. Such spatial modulation of the toroidal moment can be achieved in a domain wall in multiferroics. From optical viewpoints, the toroidal moment plays the role of a 'vector potential of light.

Since the OME effect is independent of polarization, we can use geometrical optics, which is a scalar representation, to describe the wave propagation through multiferroics. The OME effect is described by

$$
n(\vec{r})=n_{0}(\vec{r})+\alpha \hat{k} \cdot \vec{T}(\vec{r}),
$$

where $\hat{k}=\vec{k} /|\vec{k}|$ is now represented as $\hat{k}=\frac{d \vec{r}}{d s}$. Substituting the refractive index (2) into Eq. (1), we obtain the following non-linear equation,

$$
\begin{array}{r}
\frac{d}{d s}\left[n_{0}(\vec{r}) \hat{k}\right]+ \\
+\alpha \frac{d}{d s}[(\hat{k} \cdot \vec{T}(\vec{r})) \hat{k}]=\operatorname{grad} n_{0}(\vec{r}) \\
+\alpha(\hat{k} \cdot \nabla) \vec{T}(\vec{r})+\alpha \hat{k} \times \operatorname{rot} \vec{T}(\vec{r}) .
\end{array}
$$

Each term on the Eq. (3) can be interpreted as follows. The first term on the right-hand side represents a "potential force" for photons which yields a conventional Snell's law. The second term is the differential of the toroidal moment along the propagation direction, whose physical meaning is explained later. Remarkably, the third term is nothing but a Lorentz force on light with a vector potential $\vec{T}(\vec{r})$. The $\hat{k}$ dependence in the second and the third terms gives rise to non-reciprocal refraction, namely, oneway propagation.

In realistic materials, the contribution of the Lorentz force term can be observed in a domain wall(DW) of the toroidal moment. In a toroidal domain-wall(TDW) region, the toroidal moment varies as $\vec{T}=(0, B x, 0)$, which is analogous to the vector potential of a uniform magnetic field $B$ in the Landau gauge, as shown in Fig. 1 Since the toroidal moment is roughly related to the electric polarization and the magnetization as $\vec{T} \propto \vec{P} \times \vec{M}$, the toroidal moment can be reversed by the reversal of $\vec{P}$ or $\vec{M}$ by an external field. In the TDW, we have $n_{0}(\vec{r})=n_{0}$ and the equation is explicitly written as

$$
\begin{aligned}
n_{0} \ddot{x}+\alpha B \frac{d}{d s}(\dot{y} x \dot{x})-\alpha B \dot{y} & =0, \\
n_{0} \ddot{y}+\alpha B \frac{d}{d s}\left(x \dot{y}^{2}\right) & =0,
\end{aligned}
$$

where the dot denotes a derivative with respect to $s$. We assume the initial conditions to be $\left.\vec{r}\right|_{s=0}=(0,0,0)$, $\left.\hat{k}\right|_{s=0}=(\cos \theta, \sin \theta, 0)$, and we can integrate the equa-
tions;

$$
\begin{array}{r}
n_{0} \dot{x}+\alpha B \dot{y} x \dot{x}-\alpha B y=n_{0} \cos \theta, \\
n_{0} \dot{y}+\alpha B x \dot{y}^{2}=n_{0} \sin \theta .
\end{array}
$$

Then the solution to these equations is

$$
x=\frac{B \alpha}{2 n_{0} \sin \theta} y^{2}+\frac{\cos \theta}{\sin \theta} y,
$$

which is interpreted as follows. The Lorentz force term of the right-hand side on Eq. (3) is explicitly written to be $\hat{k} \times \operatorname{rot} \vec{T}=\left(\hat{k}_{y} B,-\hat{k}_{x} B, 0\right)$, which gives rise to a cyclotron motion. The second term gives $(\hat{k} \cdot \nabla) \vec{T}=\left(0,+\hat{k}_{x} B, 0\right)$ which cancels the $y$ component of the Lorentz term. Therefore the right-hand side in Eq. (3) is proportional to $\left(\hat{k}_{y} B, 0,0\right)$, leading to the parabolic solution. When the direction of the initial velocity is reversed, a sign of a curvature of the parabola is changed, which means that we have a one-way trajectory through a TDW. Because the toroidal moment is a physical field for optical waves and is related to the electric polarization and the magnetization, it has no gauge invariance and can be controlled by external fields or temperature. It means that one can design a vector potential at one's disposal by spatially modulating $\vec{P}$ or $\vec{M}$.

Let us estimate the displacement $\delta$ in Fig. 1. We put the thickness and the height of a sample to be $l$ and $h$, respectively. The displacement is roughly estimated as $\delta \sim\left(h^{2} / l\right) a$, where $a$ is the strength of the OME effect; $a=\left(n_{\rightarrow}-n_{\leftarrow}\right) / n_{0}$. In $\mathrm{GaFeO}_{3}$, these parameters are $l \sim 100 \mathrm{~nm}, h \sim 10 \mu \mathrm{m}$ and $a \sim 10^{-3}$. Using these parameters, we obtain the displacement to be $\delta \sim 1 \mu \mathrm{m}[11$, which can be observed through a microscope. A condition for large $\delta$ is to have a large amount of magnetic field and a large medium. Such a strong magnetic field can be achieved in a material with a large OME effect and a thin TDW, namely large $a$ and small $l$, respectively. A large OME effect gives rise to a large refraction, and small $l$ corresponds to a large differential of the toroidal moment, resulting in a large displacement. Here the validity of the geometrical optics should be discussed. Since the thickness of the DW in $\mathrm{GaFeO}_{3}$ is $100 \mathrm{~nm}$ which is shorter than the wavelength of the visible light, the refraction of the light would be obscure. Therefore we should use ultraviolet beam. However, a material with a DW whose thickness is larger than the wavelength of the incident light might be a good candidate to observe this non-reciprocal refraction.

As an example, we consider an experimental set up for the TDWs shown in Fig. 2 Here, we introduce the TDW as a magnetic DW with uniform electric polarization. The incident optical ray is refracted through each DW, leading to two distinct regions as contrasts in a screen. One is a dark region represented by dark blue where photons cannot reach. The other is a bright region 
characterized by a white color where both refracted light and transmitted light can reach. It means that TDWs can be observed as contrasts in a screen 12. The region in which the light propagates out of TDWs is represented by a bright blue color. The contrasts purely have their origins in the spatially varying toroidal moment. To examine whether the contrasts are really caused by the inhomogeneous toroidal moment or not, we propose following two experimental methods. One is to introduce the light from another side of the sample. There should be different contrasts from those of another side, represented by two screens 1 and 2 in Fig. 22 The other is to apply the external magnetic field $\sim 500$ Oe parallel to $z$ direction to order the magnetization. The magnetic DW and the TDW vanish, resulting in the disappearance of the contrasts. Both of these two experimental tests are needed to confirm our proposal.

The OME effect is usually very small for direct observation; $n_{\rightarrow}-n_{\leftarrow} \sim 10^{-3}$ for $\mathrm{GaFeO}_{3}|8|, \sim 10^{-6}$ for $\mathrm{Cr}_{2} \mathrm{O}_{3}$ [13], $\sim 10^{-4}$ for $\operatorname{Er}_{1.5} \mathrm{Y}_{1.5} \mathrm{Al}_{5} \mathrm{O}_{12}$ 14] and so on. To overcome the disadvantage, one can enhance the OME effect in artificial structures. In periodic structures composed of multiferroics, the OME effect can be magnified by a factor of 1000 in multiferroic gratings and photonic crystals [15]. In contrast, what we demonstrate here provides another example for a sizable OME effect. The non-reciprocal refraction obtained in this Letter is of the order of $1 \mu \mathrm{m}$, which can be observed without any use of a cavity, a photonic crystal or a patterned structure. Therefore, non-reciprocal refraction due to a toroidal moment modulation provides us with a new method to measure the OME effect.

Kida et. al. found an OME effect in a submicron patterned magnet in which inversion symmetry is artificially broken by a chevron-shaped structure [16]. The signal of the OME effect in the patterned magnet is of the order of $10^{-3}$, which is significantly large. They show that we can introduce a toroidal moment in a magnet with an artificially asymmetric structure. It may be possible that an inhomogeneous toroidal moment introduced by an artificial structure yields a Lorentz force on light.

In conclusion, we have investigated a novel nonreciprocal refraction in multiferroics, which is polarization independent. The toroidal moment, inherent in multiferroics, plays the role of vector potential for light, and its spatial variation gives optical analogue of a Lorentz force. Photons feel no Lorentz force in a conventional sense under a real external magnetic field because they have no electric charge. The toroidal moment is a physical field without gauge degrees of freedom, and can be experimentally controlled by an external field or temperature. It means that the Lorentz force can also be controlled by them. Such an interesting material as a multiferroic matter has the potential importance of a novel external field for photons; the Lorentz force obtained here is one example. This Lorentz force on light fills one of the most fundamental pieces which has been missing for photon-electron analogy.

The authors are grateful to T. Arima, M. Onoda, N. Kida, S. Murakami, H. Katsura, and Y. Tokura for fruitful discussions. K.S. is supported by a Japan Society for the Promotion of Science. This work is financially supported by a NAREGI Grant, Grant-in-Aids from the Ministry of Education, Culture, Sports, Science and Technology of Japan.

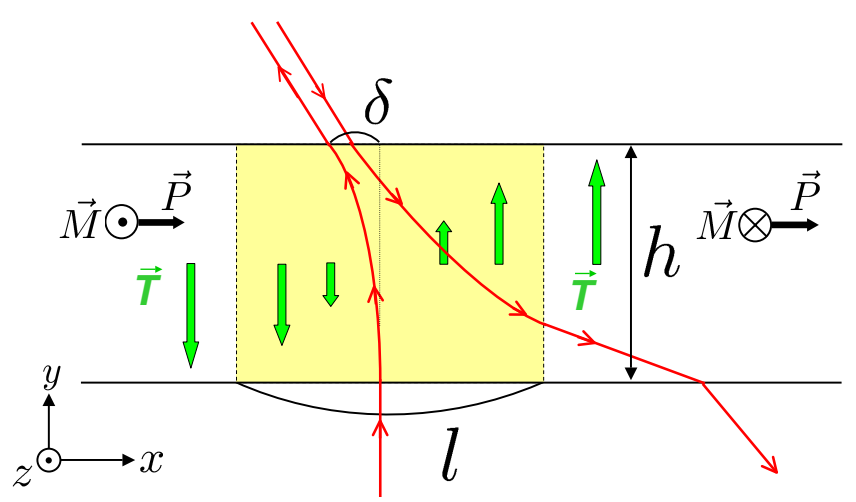

FIG. 1: Non-reciprocal refraction through a toroidal domain wall represented by a yellow box. The green arrows represent toroidal moments. Different propagation direction has different optical path, represented by the lines with red arrows.

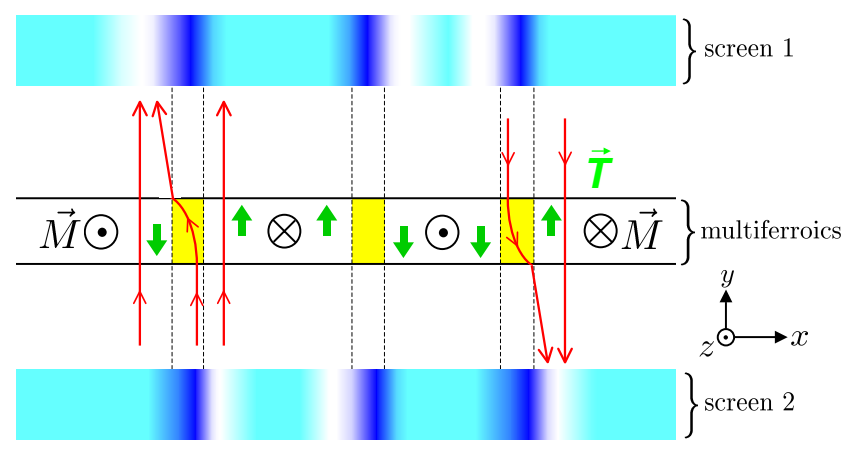

FIG. 2: Non-reciprocal refraction through some domain walls. Domain walls are observed as contrasts on a screen, represented by deep blue, bright blue, and white regions. The two screens 1 and 2 with different contrasts correspond to the incident directions, the face and the back, respectively.

* Electronic address: sawada@appi.t.u-tokyo.ac.jp

$\dagger$ Electronic address: nagaosa@appi.t.u-tokyo.ac.jp

[1] S. Datta, Electronic Transport in Mesoscopic Systems, (Cambridge University Press, 1995).

[2] M. Born and E. Wolf, Principles of Optics, 7th edition (Cambridge University Press, 1999).

[3] M. Onoda, S. Murakami and N. Nagaosa, Phys. Rev. Lett. 93, 083901 (2004). 
[4] K. Yu. Bliokh and Yu. P. Bliokh, Phys. Rev. E 70, 026605 (2004); JETP Lett. 79, 519 (2004).

[5] F. D. M. Haldane and S. Raghu, cond-mat/0503588

[6] L. D. Barron and J. Vrbancich, Mol. Phys. 51, 715 (1984).

[7] G. L. J. A. Rikken and E. Raupach, Nature 390, 493 (1997); G. L. J. A. Rikken and E. Raupach, Phys. Rev. E 58, 5081 (1998); C. Koerdt, G. Düchs and G. L. J. A. Rikken, Phys. Rev. Lett. 91. 073902 (2003); F. A. Pinheiro and B. A. van Tiggelen, J. Opt. Soc. Am. A 20, 99(2003).

[8] M. Kubota, T. Arima, Y. Kaneko, J. P. He, X. Z. Yu and Y. Tokura, Phys. Rev. Lett. 92. 137401 (2004); J. H. Jung, M. Matsubara, T. Arima, J. P. He, Y. Kaneko and Y. Tokura, Phys. Rev. Lett. 93037403 (2004); T. Arima, J. H. Jung, M. Matsubara, M. Kubota, J. P. He, Y. Kaneko and Y. Tokura, J. Phys. Soc. Japan 74, 1419 (2005).

[9] S. Murakami, R. Shindou, N. Nagaosa and A. S. Mishchenko, Phys. Rev. B 66, 184405 (2002).

[10] Yu. F. Popov, A. M. Kadomtseva, G. P. Vorob'ev, V. A. Timofeeva, D. M. Ustinin, A. K. Zvezdin and M. M. Tegeranchi, J. Exp. Theor. Phys. 87, 146 (1998).
[11] For $\mathrm{GaFeO}_{3}$, optical path in Fig. 1 is not the case. The thickness $l$ is so small in $\mathrm{GaFeO}_{3}$ that optical beam is refracted and out of a TDW. The trajectory of the beam is a parabola in a TDW and becomes a line out of a TDW. The naive estimation $\delta \sim \frac{h^{2}}{l} a$ breaks down. However, careful calculation suggests that the displacement still holds $\delta \sim 1 \mu \mathrm{m}$.

[12] These contrasts simply represent whether photons reach or not, regardless of interference due to difference in light paths of refracted one and unrefracted one. Coherent light may make the interference pattern on a screen in addition to the contrasts.

[13] B. B. Krichevtsov, V. V. Pavlov, R. V. Pisarev, and V. N. Gridnev, J. Phys. Condens. Matter 5, 8233 (1993).

[14] G. L. J. A. Rikken, C. Strohm and P. Wyder, Phys. Rev. Lett. 89, 133005 (2002).

[15] K. Sawada and N. Nagaosa, Appl. Phys. Lett. 87, 042503 (2005).

[16] N. Kida, T. Yamada, M. Konoto, Y. Okimoto, T. Arima, K. Koike, H. Akoh and Y. Tokura, Phys. Rev. Lett. 94, 077205 (2005). 